\title{
What comes first-genomic structure or function?
}

CRISPR-mediated chromatin looping provides insight into the effect of genomic structure on gene expression.

The causality dilemma has puzzled mankind since ancient times. Kevin Wang and his team at Stanford University now address a molecular equivalent of the chicken and egg question: "Does gene structure come before gene expression, or does gene expression beget structure?"

Wang had been interested in the structure versus function question since his days as a postdoctoral fellow, when he studied how noncoding RNAs stabilize active chromatin. To determine the causal link between chromatin structure and transcription, Wang asked whether he could form a loop between two loci and induce expression.

To answer this question, he first needed a method to trigger chromatin looping. Wang recalls that TALEs were the latest option for genome engineering at the time, but he did not pursue these tools, because their repetitive design proved to be too complicated for his purpose. He did not have to wait too long for a much easier approach. "When CRISPR came out, we thought it was a nice targeted method to do exactly what we wanted."

In efforts led by graduate student Stefanie Morgan, the team developed CLOuD9 (chromatin loop reorganization using CRISPR-dCas9) to establish new chromatin loops. The researchers used orthogonal Streptococcus pyogenes and Staphylococcus aureus Cas9 species fused to dimerization domains and targeted them to the chromosomal loci they wished to bring together. They induced dimerization of the Cas 9 proteins via abscisic acid (ABA), a plant hormone.

It took the team a few rounds of trial and error to optimize CLOuD9 in cultured cells and to decide on the right dimerizer; the advantage of ABA, besides its being cheap and biologically inert, is that it is part of an animal's diet. Eventually Wang wants to move the technology into animals and simply trigger dimerization via food.

The researchers first targeted the wellstudied $\beta$-globin locus in a leukemia cell line that predominantly expresses fetal $\gamma$-globin over adult $\beta$-globin. By bringing the $\beta$-globin locus together with its distal locus control region they robustly increased $\beta$-globin expression. Intriguingly, the same induced chromatin contacts in a human kidney cell line did not affect $\beta$-globin gene expression. "This is important," says Wang, "because it shows that loop formation only matters in a particular context."

When the team induced contact between the promoter of the transcription factor Oct4, essential for self-renewal of embryonic stem cells, and a distal $5^{\prime}$ enhancer in the kidney cell line, they could trigger Oct4 expression. The strength of looping dictated the level of gene expression; if Oct4 was looped together with multiple enhancers, gene expression increased by several hundred fold. "We are still trying to figure out what the rules are," says Wang. "This will tell us whether we can come up with an algorithm that tells you how many contacts you need to improve gene expression."

Withdrawal of ABA after several hours of induction led to a reversal of the chromatin contacts, and gene expression of the target genes ceased. But there was a caveat. After days of ABA-induced dimerization, the chromatin contacts proved to be stable, and elevated levels of gene expression persisted even after ABA removal. The researchers determined that two RNA helicases were responsible for maintaining chromatin topology. This points to a new regulatory mechanism that awaits further follow up.

Wang also plans to use CLOuD9 to turn genes off by looping a repressive domain to an active gene. He predicts that the aberrant expression of the myc oncogene in cancer cells will make a suitable first target.

Forced chromatin contacts will also help researchers address another question in the field, namely, whether topology associating domains (TADs) are functional boundaries. Wang plans to look at the effect of interTAD and intra-TAD loops at the genome scale to provide some answers.

What CLOuD9 has already made clear: genome structure precedes function. Nicole Rusk

\section{RESEARCH PAPERS}

Morgan, S.L. et al. Manipulation of nuclear architecture through CRISPR-mediated chromosomal looping. Nat. Commun. http://dx.doi.org/10.1038/ ncomms15993 (2017). 